\title{
Estudo teórico das propriedades óticas não linea- res de nanopartículas metálicas
}

Filho, J. B. M.

Escola Politécnica de Pernambuco Universidade de Pernambuco

50.720-001 - Recife, Brasil joaomonteirof@gmail.com

\author{
Malagón, L. A. G. \\ Escola Politécnica de Pernambuco \\ Universidade de Pernambuco \\ 50.720-001 - Recife, Brasil \\ lagomezma@poli.br
}

Resumo Foram estudadas as propriedades óticas não lineares de materiais contendo nanopartículas de prata $e$ de ouro utilizando alguns modelos recentes. Fator de campo local e susceptibilidade de terceira ordem foram calculados em relação ao tamanho das nanopartículas e em relação às características do laser incidente como intensidade e comprimento de onda. Os resultados obtidos descrevem o comportamento experimental reportado na literatura.

\footnotetext{
Abstract The nonlinear optical properties of nanosized silver and gold materials were studied by the use of some recent mathematical models. Local field factor and third order susceptibility were calculated in order to verify their relation with incident laser's characteristics like it's intensity and wavelength. The obtained results describe the experimental behavior reported in the literature.
} 


\section{Introdução}

A ótica não linear é o ramo da ótica que estuda a resposta de materiais a excitações luminosas intensas. Essa resposta é função do meio excitado através de propriedades como a susceptibilidade, por exemplo. O cálculo das propriedades óticas não lineares de materiais contendo nanopartículas metálicas tem sido um problema bastante atacado, com resultados interessantes. Esse trabalho baseiase em modelos recentes e dados para cálculo e comparação dos resultados de propriedades óticas não lineares de materiais contendo nanopartículas metálicas de prata e de ouro fazendo uso de ferramentas computacionais de análise de dados como o Origin e o ambiente multifuncional do Matlab com o objetivo da validação, incluindo algumas correções, dos modelos utilizados.

\section{Modelo Matemático}

Assume-se um composto de topologia onde as partículas que, por simplificação, serão consideradas esféricas, estão distribuídas aleatoriamente num meio hospedeiro. Sendo a o raio da nanopartícula, $b$ a distância característica e $\lambda$ o comprimento de onda da luz incidente, vale o modelo de Maxwell-Garnett se vale a condição $\mathrm{a}<<\mathrm{b}<<\lambda$ e se a fração de volume, , do metal em relação ao hospedeiro for muito pequena. O modelo de Maxwell-Garnett é um EMA (Effective Medium Aproximation), um tipo de modelo que tenta prever as propriedades de um sistema a partir das propriedades de seus constituintes. De acordo com esse modelo, vale a equação:

$$
\frac{\varepsilon-\varepsilon_{h}}{\varepsilon+2 \varepsilon_{h}}=p \frac{\varepsilon_{m}-\varepsilon_{h}}{\varepsilon_{m}+2 \varepsilon_{h}}
$$

em que $\varepsilon, \varepsilon_{h}$ e $\varepsilon_{m}$ são as constantes dielétricas do material, do meio hospedeiro e do metal constituinte das nanopartículas, respectivamente.

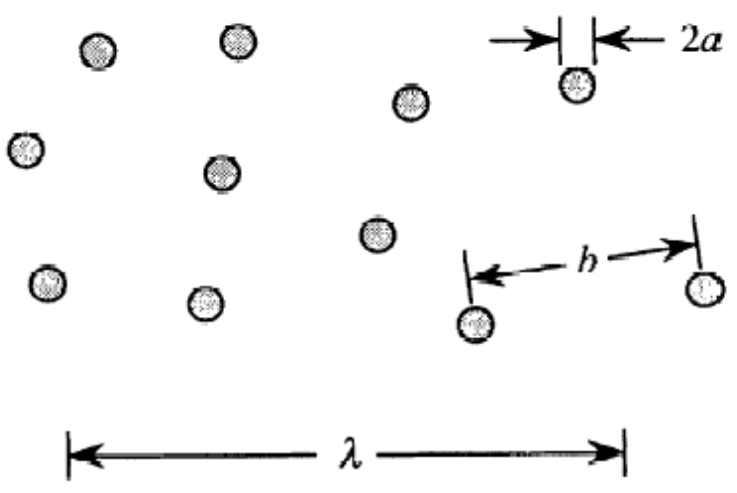

Fig 1.Topologia assumida para as soluções estudadas.

Existe uma relação entre a parte imaginária da constante dielétrica e o coeficiente de absorção. A relação pode ser escrita assim:

$$
\alpha=\frac{18 \pi p}{\lambda} \varepsilon_{h}^{3 / 2} \frac{\varepsilon_{m}^{\prime \prime}}{\left(\varepsilon_{m}^{\prime}+2 \varepsilon_{h}\right)^{2}+\varepsilon_{m}^{\prime \prime}}
$$

Assumindo que apenas as nanopartículas apresentam efeitos não lineares, podemos assim obter a expressão para o cálculo de qui3:

$$
\chi_{e f f}^{(3)}=p f^{2}|f|^{2} \chi_{m}^{(3)}
$$

O modelo do gás de elétrons degenerado é um modelo recente que foi desenvolvido tomando como situação de análise um elétron num poço esférico de potencial infinito com raio R. Este modelo tem como ponto de inovação o fato de considerar o Hamiltoniano da interação entre o elétron e o campo eletromagnético numa forma diferente. Modelos antigos utilizavam o Hamiltoniano na forma:

$$
\widehat{V}=-\frac{e}{m c} \hat{p} \cdot A+\frac{e^{2}}{2 m c^{2}} A^{2}
$$

onde p é o momentum do elétron e A é o vetor potencial.

O modelo citado escreve-o na forma: 


$$
\widehat{V}=-\widehat{d} \cdot E
$$

onde E é o campo elétrico atuante.

Ficou provado na ref. [2] que as formas só são equivalentes para excitações na freqüência de ressonância. $O$ último modelo citado é útil para o cálculo de $X^{(3)}$ das nanopartículas metálicas, através da equação:

$$
\begin{gathered}
\chi_{m}^{(3)}=\frac{2}{15}\left(\frac{e^{2} n}{m \omega^{2}}\right)\left(\frac{2 \pi e a}{h \omega}\right)^{2} \frac{\Gamma_{2}}{\Gamma_{1}}\left\{F_{3}-i\left[\frac{2 \Gamma_{2}}{\omega} F_{3}+\right.\right. \\
\left.\left.\left(\frac{\omega}{2 \Gamma_{2}}\right)^{2}\left(\frac{v_{f}}{a \omega}\right)^{5} g_{3}\right]\right\}
\end{gathered}
$$

onde $\omega$ é a freqüência angular da excitação; $\Gamma_{1}$ e $\digamma_{2}$ representam as taxas de relaxação populacional e de coesão, respectivamente, e foram parâmetros de ajuste no desenvolvimento do trabalho; $F_{3}$ e $g_{3}$ são algumas das constantes que foram calculadas através do ajuste da constante dielétrica aos dados experimentais.

O objetivo principal do trabalho foi o cálculo da susceptibilidade de terceira ordem para materiais contendo nanopartículas de ouro.

Para o cálculo dessa propriedade, houve a necessidade de encontrar o valor de algumas outras.

Podemos utilizar a Equação (3) para o cálculo da susceptibilidade, mas para isso precisamos conhecer o valor do fator de campo local, e da susceptibilidade dos metais envolvidos.

O fator de campo local é dado por, de acordo com a ref.[10]:

$$
f=\frac{3 \varepsilon_{h}}{\varepsilon_{m}+2 \varepsilon_{h}}
$$

Para a prata, a constante dielétrica pode ser escrita (incluindo correções de intensidade e de tamanho da nanopartícula) na forma:

$$
\varepsilon_{m}=\varepsilon_{\text {bulk }}+i g_{1}\left(\frac{4 \pi e^{2} n}{m \omega^{2}}\right) \frac{v_{f}}{a \omega}+12 \pi \chi_{m}^{(3)}|f|^{2} I_{0}
$$

onde $g_{1}$ é uma das constantes determinadas pelo ajuste da constante dielétrica; $\varepsilon_{\text {bulk }}$ é a constante dielétrica para uma "porção extensa" do material e $v_{f}$ é a velocidade de Fermi.

Utilizando as Equações (7) e (8), podemos chegar a:

$$
A|f(\omega)|^{6}+B|f(\omega)|^{4}+C|f(\omega)|^{2}+D=0
$$

onde:

$$
\begin{gathered}
A=c^{2}+d^{2} \\
B=2(a c+b d) \\
C=a^{2}+b^{2} \\
D=-9 \varepsilon_{h}^{2}
\end{gathered}
$$

$\mathrm{e}$

$$
\begin{gathered}
a=\operatorname{Re}\left(\varepsilon_{m}^{0}\right)+2 \varepsilon_{h} \\
b=\operatorname{Im}\left(\varepsilon_{m}^{0}\right) \\
c=I_{0} 12 \pi \operatorname{Re}\left(\chi_{m}^{(3)}\right) \\
d=I_{0} 12 \pi \operatorname{Im}\left(\chi_{m}^{(3)}\right)
\end{gathered}
$$

A equação (9) pode ser resolvida numericamente. Com os resultados em mãos, calculasse a susceptibilidade de terceira ordem para o material.

Para o ouro, a expressão utilizada para a constante dielétrica foi:

$$
\varepsilon_{m}=1-\frac{\omega_{p f}^{2}}{\omega^{2}+i \frac{\omega}{\tau_{f}}}+\frac{\omega_{p b}^{2}}{\omega_{0}^{2}-\omega^{2}-i \frac{\omega}{\tau_{b}}}
$$

onde $\omega_{p f}, \omega_{p b}$ e $\omega_{0}$ são freqüências características das correções efetuadas para se chegar à expressão (10). As constantes $t_{f}$ e $t_{b}$ são análogas a $; \Gamma_{1}$ e $\Gamma_{2}$. Todas essas constantes foram obtidas por ajuste da expressão (10) a dados experimentais da ref. [3].

\section{Tratamento Numérico}

Os cálculos iniciais tinham o objetivo da determinação de algumas constantes que seriam utilizadas durante o restante do trabalho. Para a prata, foi utilizada a Equação (8), excluindo os efeitos de intensidade, para ajuste a dados experimentais da ref. [3], utilizando a ferramenta de ajuste do Origin. Para o ouro, foi feito o mesmo procedimento, mas foi utilizada para o ajuste a Equação (10).

Algumas equações complexas precisaram ser resolvidas. A Equação (9) consiste de um polinômio de sexta ordem no módulo do fator de campo local, onde os coeficientes são funções de características como o comprimento 
de onda, a intensidade da luz incidente, o raio das nanopartículas etc. Para resolver esta equação, implementou-se um algoritmo que calcula os coeficientes para cada variação de uma das variáveis citadas, mantendo-se todas as outras constantes. Computam-se os resultados das raízes. O problema, a partir deste ponto, é que cada solução encontrada consistia em seis raízes do polinômio. Como a Equação (9) é uma equação polinomial no módulo do fator de campo local, só são válidas as raízes reais e positivas. Algoritmos de testes foram desenvolvidos para avaliar intervalos do domínio onde apenas uma das raízes seria real e positiva, evitando problemas de resultados múltiplos. Dessa forma, foi possível computar o módulo do fator de campo local em função do comprimento de onda, da intensidade incidente e do raio da nanopartícula.

Para o cálculo de $X_{\text {eff }}^{(3)}$, pode ser utilizada diretamente a Equação (3).

\section{Resultados obtidos e discussão}

Para as constantes envolvidas nas equações propostas, foram feitos ajustes com dados contidos na ref. [3]. Vêemse nas figuras que seguem os ajustes feitos:

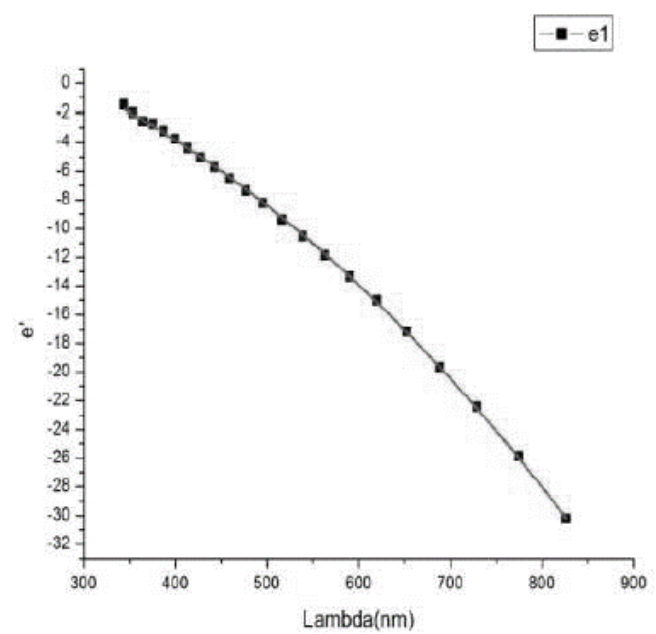

Fig 2. Parte real da constante dielétrica da prata em função do comprimento de onda.

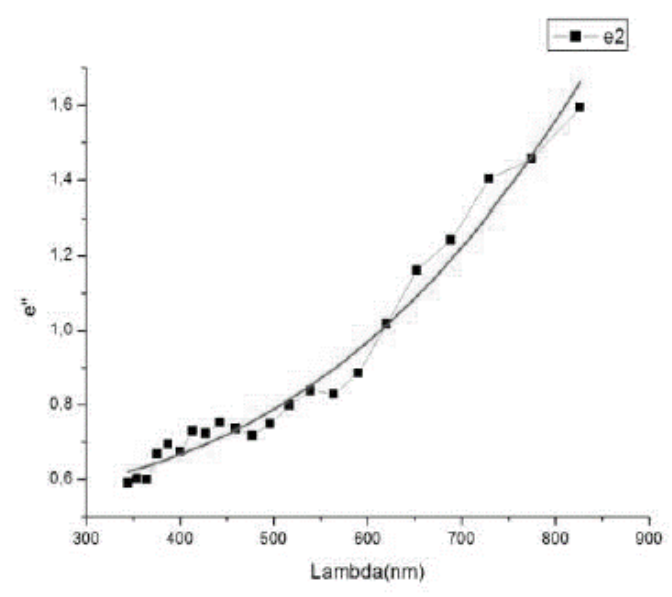

Fig 3. Parte imaginária da contante dielétrica da prata em função do comprimento de onda.

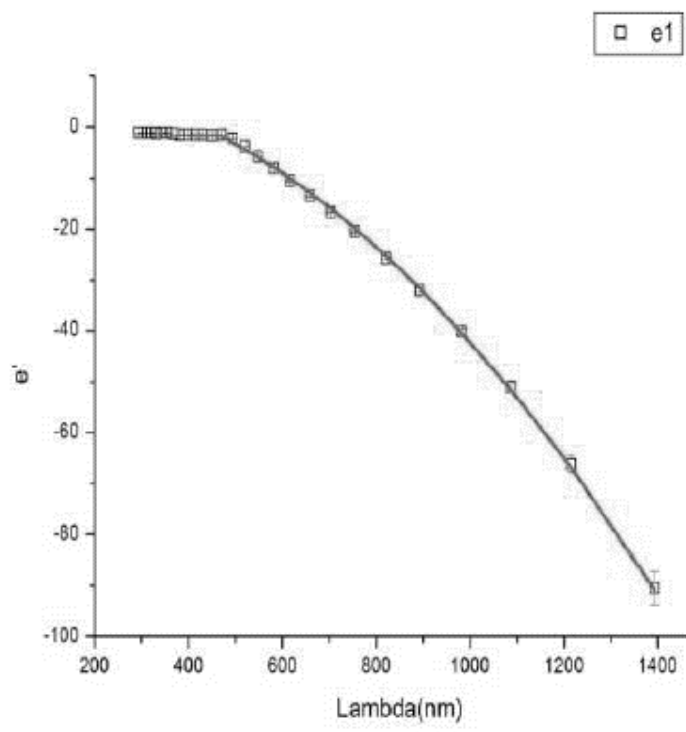

Fig 4. Parte real da constante dielétrica do ouro em função do comprimento de onda. 


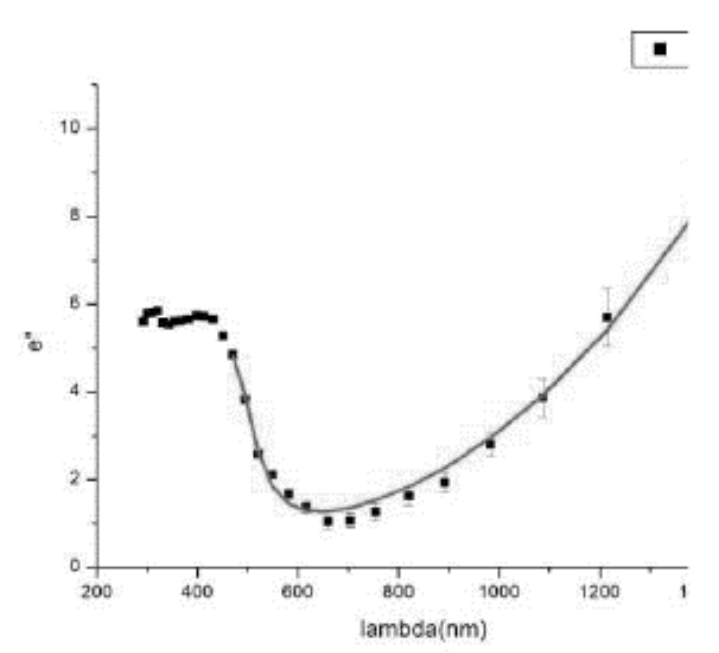

Fig 5. Parte imaginária da constante dielétrica do ouro em função do comprimento de onda.

Nas Figuras 1 e 2, foram ajustadas as partes real e imaginária da constante dielétrica da prata de acordo com a Equação (8), porém sem levar em consideração efeitos de intensidade incidente. A ferramenta de ajuste de curvas do Origin foi utilizada para o cálculo das constantes citadas. Nas Figuras 3 e 4, foi realizado o mesmo procedimento para o ouro, porém, de acordo com a Equação (10).

\section{Para a prata temos:}

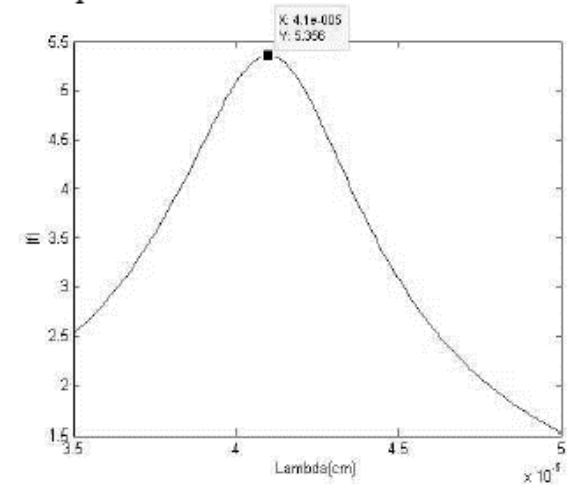

Fig 6. Módulo do fator de campo local em função do comprimento de onda.

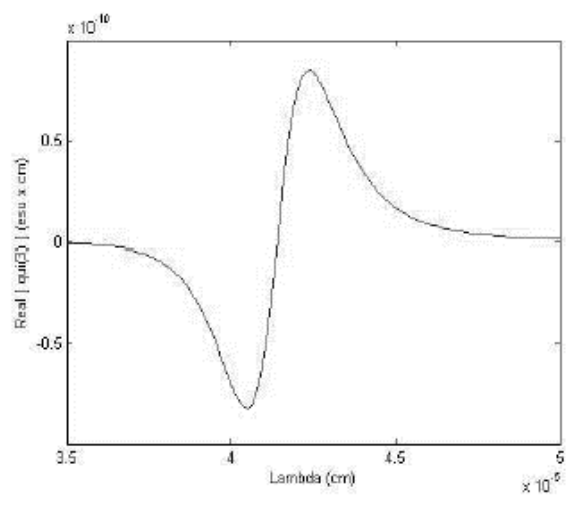

Fig 7. Parte real da susceptibilidade em função do comprimento de onda.

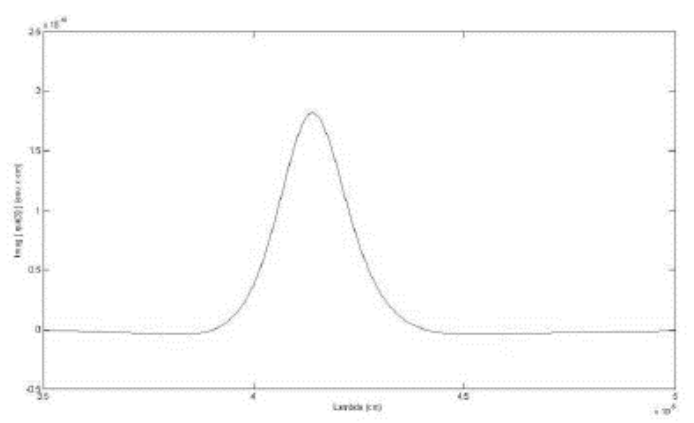

Fig 8. Parte imaginária da susceptibilidade em função do comprimento de onda.

As Figuras 5, 6 e 7 apresentam resultados para cálculos com intensidade de $1 \mathrm{Mw} / \mathrm{cm}^{2}$ e raio das nanopartículas igual a 3 nanometros. Na Figura 5, podemos observar um pico. Como pode ser visto ma ref. [1], para alguns valores do raio da nanopartícula e de intensidade incidente, para um mesmo comprimento de onda podem ser obtidos três valores do módulo do fator de campo local. Para evitar situações como essa, algorimos de teste foram feitos e os resultados só foram calculados para situações de comportamento como o mostrado na Figura 5.

Para o ouro, temos: 


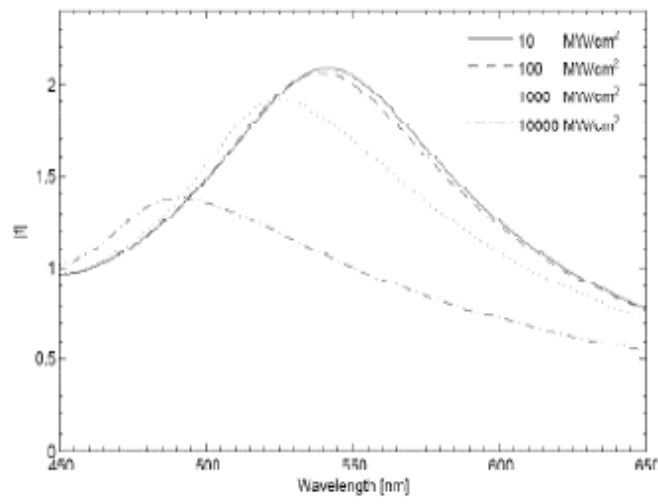

Fig 9.Módulo do fator de campo local em função do comprimento de onda.

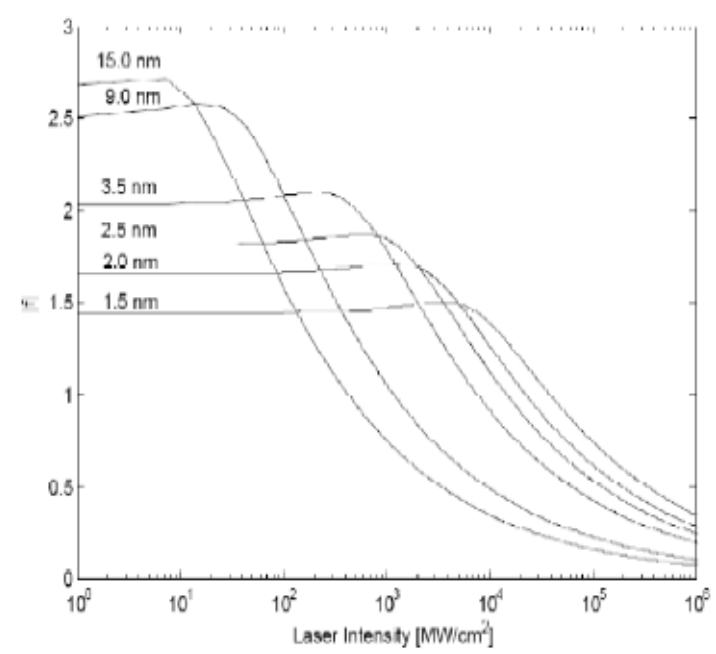

Fig 10. Módulo do fator de campo local em função da intensidade da luz incidente.

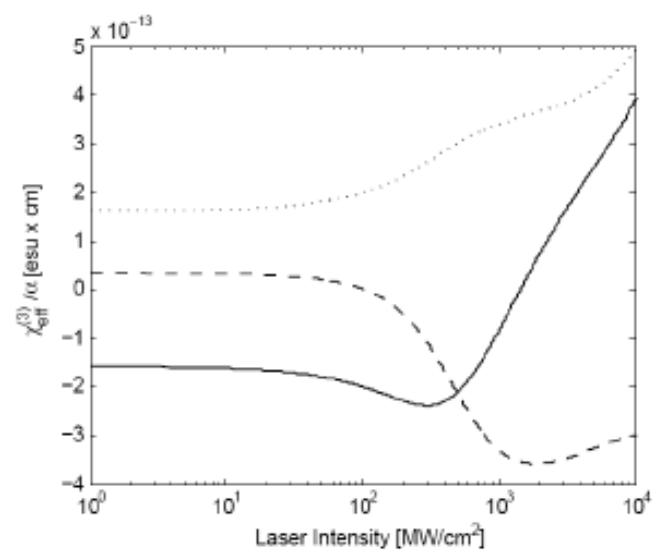

Fig 11. Partes real, imaginária e módulo da razão entre a susceptibilidade e o coeficiente de absorção para a mistura em função da intensidade incidente.

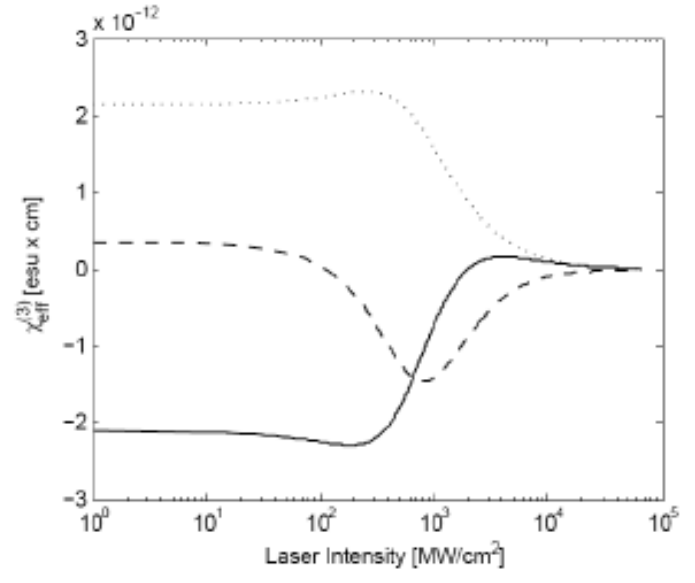

Fig 12. Partes real, imaginária e módulo da susceptibilidade para a mistura em função da intensidade incidente.

A figura 8 traz a relação entre o módulo do fator de campo local e o comprimento de onda. É importante destacar o deslocamento à esquerda do valor de pico da relação com o aumento da intensidade.

A Figura 9 mostra a relação entre o módulo do fator de campo local e a intensidade incidente para vários raios das nanopartículas. Vê-se claramente que a partir de certa intensidade a curva começa a decrescer e também que isso acontece mais "rapidamente" para partículas com raio maior. Para valores altos de intensidade começa a manifestar-se o efeito da saturação, que é a origem para efeitos de bi estabilidade, ou seja, possibilidade de mais de valor para o módulo do fator de campo local. Essa região não foi utilizada nos cálculos.

\section{Conclusões}

Pela coerência entre os resultados obtidos pelo trabalho em questão e os resultados expostos em 1, ficam validados os procedimentos de cálculo para solução das equações apresentadas para a prata. Para o ouro, os resultados obtidos foram os esperados de acordo com a análise inicial (análise para prata). Além disso, tiveram comportamento análogo ao que havia sido encontrado anteriormente, mesmo após as alterações realizadas no modelo (alteração no cálculo da constante dielétrica). Os fatos citados indicam uma diretriz eficaz na análise que, infelizmente, não fica comprovada por comparação com dados de experimentos, ficando a possibilidade de comparações para um trabalho posterior. 


\section{Referências}

[1] Vladimir P. Drachev, Andrei K. Buin, Heinz Nakotte, Vladimir M. Shalaev, "Size Dependent qui(3) for Conduction Electrons in Ag Nanoparticles", Nano letters, 2004;

[2] S. G. Rautian, "Nonlinear saturation spectroscopy of the degenerate electron gas in spherical metallic particles", Zh. E ' ksp. Teor. Fiz., 1997;

[3] P. B. Jonhson, R.W. Crhisty, "Optical constants of noble metals", Phys. Review Letters, 1972;

[4] B. Karthikeyan, Jinto Thomas, Reji Philip, “Optical nonlinearity in glass-embedded silver nanoclusters under ultrafast laser excitation", Chemical Physics Letters, 2005;

[5] J.E. Sipe, Robert W. Boyd, "Nonlinear susceptibility of composite optical materials in the Maxwell Garnett model”, Physical review, 1992;

[6] R. Ruppin, "Evaluation of extended MaxwellGarnett theories", Optics Communications, 2000;

[7] Robert W. Boyd, "Nonlinear Optics", Hardcover, 2008;

[8] Vladimir P. Drachev, Uday K. Chettiar, Alexander V. Kildishev, Hsiao-Kuan Yuan, Wenshan Cai, and Vladimir M. Shalaev, "The Ag dielectric function in plasmonic metamaterials", Optical Society of America, 2004;

[9] R. M. Eisberg, "Fundamentos da Física Moderna", Guanabara, 1979;

[10] J. D. Jackson, "Eletrodinâmica Clássica”, Guanabara, 1983;

[11] J. B. Marion, "Classical Electromagnetic Radition”, Academic Press, 1965;

[12] P. A. Tipler, "Física V.4”, Guanabara, 1991; 